\title{
WiFi Network Planning and Intra-Network Interference Issues in Large Industrial Warehouses
}

\author{
David Plets ${ }^{1}$, Emmeric Tanghe ${ }^{1}$, Alec Paepens ${ }^{2}$, Luc Martens ${ }^{1}$, Wout Joseph ${ }^{1}$, \\ ${ }_{1}^{1}$ Minds-INTEC/WiCa, Ghent University, Ghent, Belgium \\ ${ }^{2}$ Volvo IT, Ghent, Belgium \\ david.plets@intec.ugent.be
}

\begin{abstract}
Industrial WiFi network planning for a very large industrial hall is performed and results are compared for two planning approaches, namely an experimental site survey and a software-based automated planning. A software-automated process and a site survey yield comparable results. Reliable results can be obtained using an automated network planner with much less effort than when executing a time-consuming classical site survey. In this way, significant cost reductions can be obtained. Further, it is shown that very strict network planning requirements can lead to a large amount of installed access points (APs), which may drastically increase intra-network interference, even after executing a frequency planning algorithm. Network planning based on high throughput requirements and the use of large link budget margins significantly increases the amount of interference in the network.
\end{abstract}

Index Terms-WiFi, propagation, measurement, WLAN, network planning, interference, throughput, performance, coverage, frequency planning, channel.

\section{INTRODUCTION}

In recent years, data communication has more and more shifted from wired to wireless, thanks to the lower installation cost of wireless deployments and the inherent possibility for client mobility. Despite security concerns and the use of dedicated protocols for industrial applications, wireless networks are now also more and more being used in shop floors, warehouses, or industrial environments in general. From a network planning perspective, the deployment of Wireless Local Area Networks (WLANs) in industrial environments is often challenging [1], particularly when a lot of metal is present and when the area that needs wireless coverage is very large. In practice, many companies order site survey studies, i.e. fully measurement-based coverage designs, to determine the number and location of required APs. However, for large environments, this can be a very time-consuming and very costly task. Moreover, WiFi deployments in large environments are often prone to intra-network interference due to the large number of APs that are installed. In this paper, the performance of an automated network planning tool combined with a short 1-day measurement campaign is compared to that of a site survey. The models that are used for the network planning are based on the results of a small and simple path loss measurement campaign. Further, it is discussed how the choice of network planning parameters influences the amount of possible interference in the network.

In Section II, the considered environment, the automated planning process, and the site survey process are presented. Section III discusses how network planning impacts intranetwork interference. Section IV compares the results of an automated network planning with those of a site survey and assesses the interference in the network. Finally, in Section V, the main results of this paper are summarized.

\section{CONFiguration}

\section{A. Environment}

Fig. 1 shows the considered environment, a large factory warehouse of 415 by 200 meters. Each of the rectangular structures represents one the 224 installed warehouse racks. Fig. 2 shows such rack, consisting of a metal frame. When filled, it contains wooden boxes with metal components inside. The height of each rack is $9 \mathrm{~m}$.

\section{B. Network planning algorithm}

The automated network planning will be performed with the WiCa Heuristic Indoor Propagation Prediction (WHIPP) tool, a wireless indoor network planning toolbox developed within the Wireless \& Cable group [2]. It allows predicting network coverage for WiFi, Zigbee, or Universal Mobile Telecommunication Systems (UMTS) and Long Term Evolution (LTE) femtocells. Another feature is an automatic network design algorithm, which optimally places APs on a floor plan, based on user-defined throughput requirements in the different rooms [2]. The WHIPP tool allows the user to choose from different available path loss models (e.g., the free-space model, the IEEE 802.11 TGn model [3],...) or to create an own pathloss model for the predictions. For this particular industrial environment, a dedicated path-loss model will be constructed.

1) Path-loss model formula: The proposed model is a oneslope model that accounts for the rack attenuations along the direct ray between transmitter Tx and receiver Rx. Mathematically, it can be expressed as follows:

$$
P L[d B]=P L_{0}+10 \cdot n \cdot \log _{10}(d)+\sum_{i} L_{R_{i}}+\chi,
$$

with $P L_{0}[\mathrm{~dB}]$ the path loss at a reference distance of $1 \mathrm{~m}$ (under the absence of any racks), $\mathrm{n}[-]$ the path-loss exponent, 


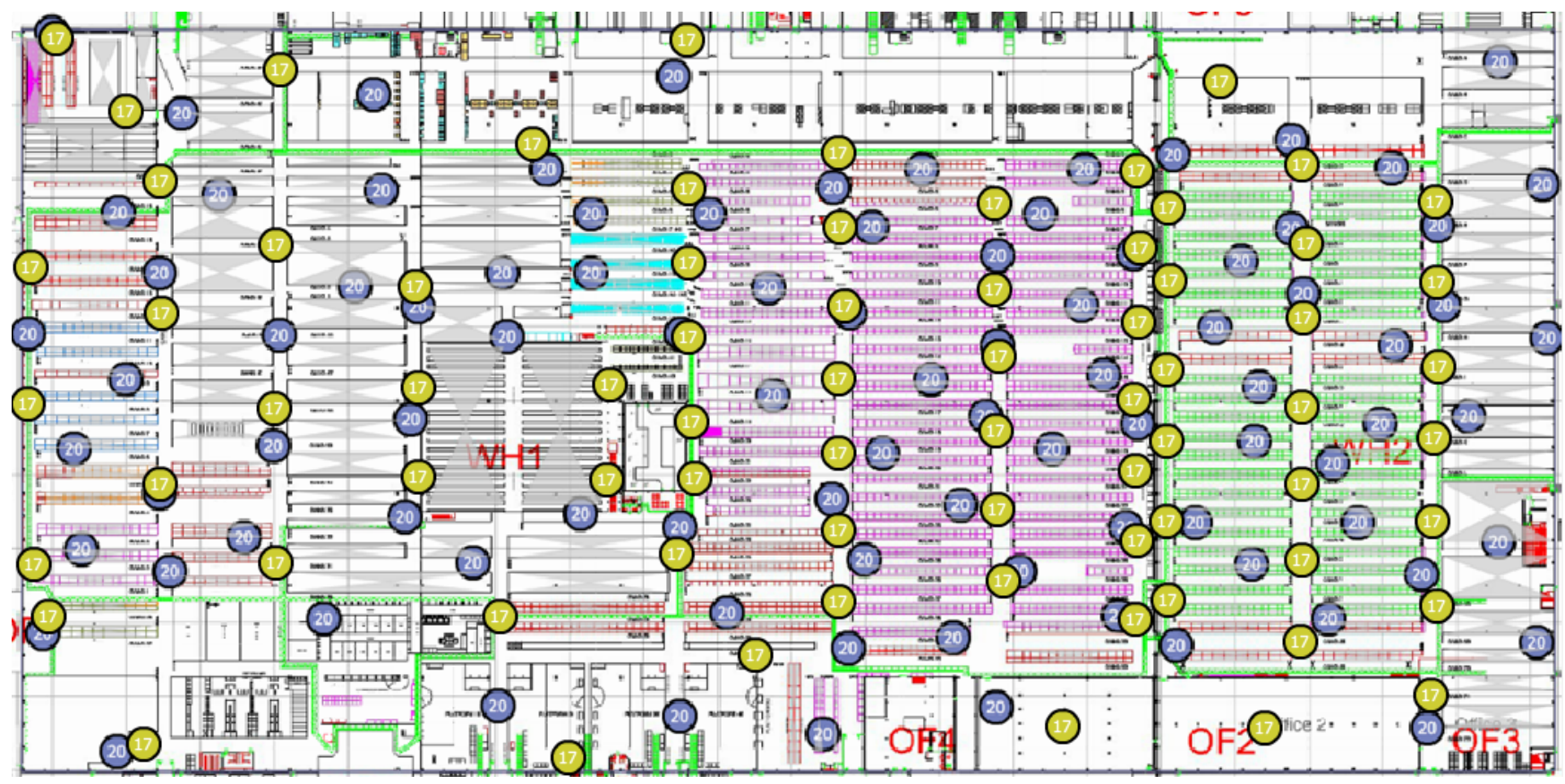

Fig. 1: Map of warehouse environment $(415 \mathrm{~m} \mathrm{x} 200 \mathrm{~m}$ ) with indication of a network design results for automated network planning (purple-dot APs) and for site survey (yellow-dot APs). The AP Equivalent Isotropically Radiated Power (EIRP) $(20 \mathrm{dBm}$ or $17 \mathrm{dBm})$ is indicated inside the dots, racks are indicated with different colourful rectangular structures with a light-grey crossed area inside.

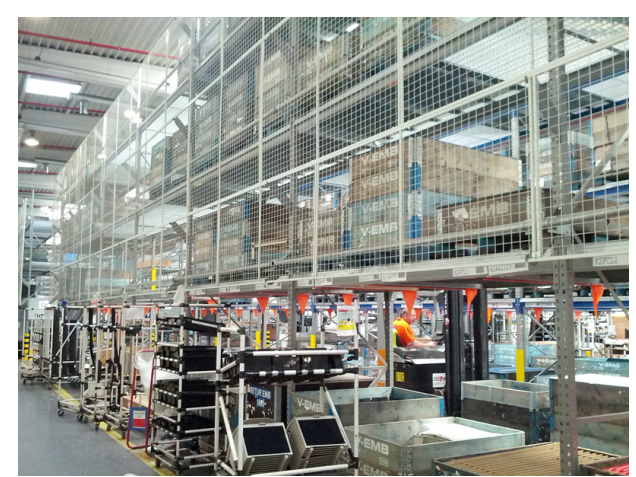

Fig. 2: Warehouse rack.

$L_{R_{i}}$ [dB] the loss of each rack $R_{i}$ that is crossed by the direct ray between $\mathrm{Tx}$ and $\mathrm{Rx}$, with a summation over all racks $R_{i} . \chi[\mathrm{dB}]$ represents the statistical variation around the model, follows a zero-mean lognormal distribution and has a standard deviation $\sigma_{\chi}$. The model is three-dimensional in a sense that it accounts for the height of the racks, as shown in Fig. 3. Although a rack might be in between Tx and Rx, its attenuation is only added in the sum when the rack is high enough to actually obstruct the direct ray: the lowest $\mathrm{Rx}$ of the two is obstructed, the highest has a line-of-sight relation with the Tx.

2) Determination of path-loss model parameters and rack attenuation: In order to determine the unknown path-loss model parameters $P L_{0}, \mathrm{n}$, and $L_{R_{i}}$, a path loss measurement campaign at $2.4 \mathrm{GHz}$ along five tracks was conducted. The

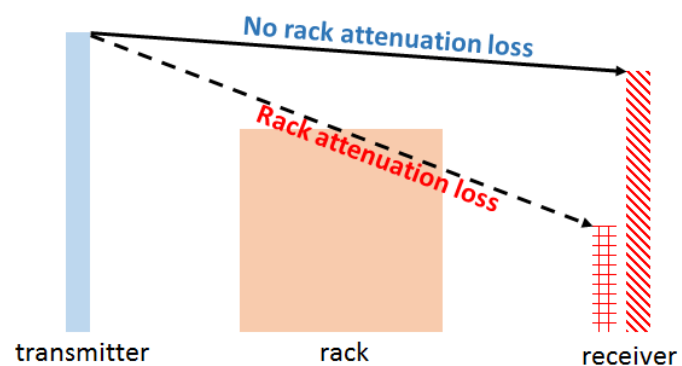

Fig. 3: Side view of example layout to illustrate how rack attenuations are accounted for.

resulting values for $P L_{0}, \mathrm{n}$, and $L_{R_{i}}$ equal $46.91 \mathrm{~dB}, 1.96$, and $4.6 \mathrm{~dB}$. The standard deviation $\sigma_{\chi}$ around the model equals $2.39 \mathrm{~dB}$. The obtained model corresponds well to the free-space model $\left(P L_{0}=40 \mathrm{~dB}, \mathrm{n}=2\right.$ at $\left.2.4 \mathrm{GHz}\right)$, with added rack attenuations.

3) Network planning requirements: The network will be planned for the $2.4 \mathrm{GHz}$ band, based on an IEEE 802.11 $\mathrm{b} / \mathrm{g}$ reference receiver. To allow video streaming to and from mobile devices (e.g., mounted on an automated guided vehicle (AGV) or used within a forklift truck), a capacity requirement of $54 \mathrm{Mbps}$ is set out, corresponding to a received signal power of $-68 \mathrm{dBm}$ [4]. The APs are installed at a height of $6 \mathrm{~m}$ above ground level with an Equivalent Isotropically Radiated Power (EIRP) of $20 \mathrm{dBm}$, and the receiver devices are assumed to be at a height of $1.3 \mathrm{~m}$, similar values as for which the path-loss 
model was constructed. From the $\sigma_{\chi}$ value of $2.39 \mathrm{~dB}$, a $95 \%$ shadowing margin of $3.92 \mathrm{~dB}$ for the link budget is derived. A temporal fading margin of $5 \mathrm{~dB}$ is assumed to account for passing machinery, people, forklift trucks,...

\section{Site survey}

In the environment depicted in Fig. 1, also a site survey was conducted to determine the number and locations of required APs, independently from the automated network planning process described in the previous section. The site survey is conducted as follows. An AP is put up on a tripod at the desired height, i.e. $6 \mathrm{~m}$. Then, a human user walks around with a handheld AirMagnet Survey Pro WLAN tester to determine the received power levels. During the measurement, an active wireless connection with the AP under test is established. By systematically moving the AP to new places over the whole area, a network is built that covers the entire area, by combining the coverage ranges of each individual AP. The required signal level for the site survey was set at $-67 \mathrm{dBm}$.

\section{INTRA-NETWORK INTERFERENCE IN NETWORK PLANNING}

In very large environments, a lot of APs can be required to meet the throughput requirements. When a wireless user receives from multiple APs at the same time a power above the noise floor (here defined as being within an AP's detection range), its achieved throughput can be significantly reduced. Frequency planning algorithms assign a frequency channel to each of the APs in a network, with the goal to reduce interference between nearby APs. Given the fact that three non-overlapping frequency bands are available for WiFi usage, the application of such algorithm will result in a network where approximately only a third of the total number of APs will be operating on the same channel. However, this can still be a large number when the network is large. The coverage zone of an AP will here be assumed as the zone where the received power is above the threshold $\mathrm{P}_{\mathrm{TP}}$ corresponding with the intended throughput (e.g., $-68 \mathrm{dBm}$ ). The interference zone is the zone where the observed power at the receiver is below $\mathrm{P}_{\mathrm{TP}}(-68 \mathrm{dBm})$, but above the noise floor detection threshold $\mathrm{P}_{\text {det }}$ (e.g., $-95 \mathrm{dBm}$ ). A wireless user in the interference zone of an AP will -in case of a correct network planning- be in the coverage zone of another AP, but its maximal throughput will be reduced due to being also in the detection range of another AP. The larger the set of all interference zones of all installed APs, the more interference wireless users will experience. Hence, three important factors influence the wireless performance.

Throughput power threshold $\mathbf{P}_{\mathbf{T P}}$ - the higher the power threshold $\mathrm{P}_{\mathrm{TP}}$ corresponding with the required throughput, the larger the area where an AP's observed power is out of the coverage range (here $<-68 \mathrm{dBm}$ ), but within the detection range $\left(>\mathrm{P}_{\text {det }}\right.$, here $\left.-95 \mathrm{dBm}\right)$. Fig. 4A indeed shows that a higher $\mathrm{P}_{\mathrm{TP}}$ drastically increases the zone where an AP will be interfering, compared to a deployment with a low
$\mathrm{P}_{\mathrm{TP}}$ (Fig. 4B). Due to the the small coverage range for higher throughputs (A), more APs will be needed to cover the considered environment, leading to more interfering APs. At the same time, the interference zone (the area from the edge of the coverage zone up to where the received power drops below $\mathrm{P}_{\text {det }}$, red zone in Fig. 4) of each AP increases. This shows that large-scale networks designed for high throughputs are more likely to suffer from intra-network interference, which will actually limit the achievable maximal throughput. Further, due to the exponentially decaying relationship between $\mathrm{P}_{\mathrm{TP}}$ and the range of an AP, the number of required (and thus also the number of possibly interfering) APs increases exponentially with an increasing power threshold.

Added margin in link budget - in traditional network planning, shadowing and fading margins are incorporated into the link budget to account for spatial and temporal variations of the signal. This corresponds to an increase of the required $\mathrm{P}_{\mathrm{TP}}$. This will further reduce the assumed coverage range of an AP and increase the number of interfering APs that will cause powers within the detection threshold. In particular, interference margins are sometimes added to the link budget to account for the influence of intra-network interference. In large networks however, such margin might in reality do the opposite of what it is designed for and might lead to increased interference.

Power decay profile - when the received power decays rapidly with the distance to the transmitter, an AP's coverage zone will be smaller, more APs will be required to provide the required coverage, and more interference can be expected. However, a rapidly decaying power will not only limit the coverage zone of an AP, but also its interference zone. Fig. 5 shows the coverage and interference zones for four different path loss models and thus, different power decay profiles. Fig. 5 shows that not only the slope of the power decrease, but also the location of the intercept (i.e., the distance from the AP at which the received power drops below $\mathrm{P}_{\mathrm{TP}}$ (plus margins)) matters. From an interference-point-of-view, a far intercept and a steep slope (situation D) is preferable: this increases the coverage zone, while limiting the interference zone. Situations A and C (far intercept and gentle slope, or near intercept and steep slope) are worse than situation $\mathrm{D}$, but better than situation B (near intercept and gentle slope). In situation B, APs have a low coverage range, so many APs are needed, but each of them is interfering up to far away due to the slow power decay.

\section{RESULTS}

Fig. 1 shows the resulting network planning based on the site survey and on the automated network planning. Fig. 1 and Table I shows that the site survey design yields a network with 73 APs (indicated with yellow dots) the automated design with the WHIPP tool yields a network with 92 APs (purple dots).

\section{A. Comparison automated planning vs. site survey}

The main difference between the automated planning and the site survey is that in the site survey, no margins are 

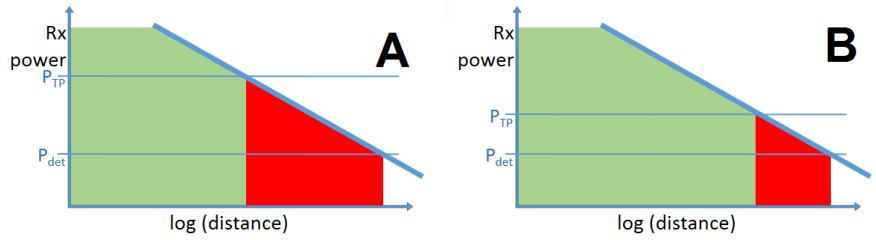

Fig. 4: Coverage zone (green) and interference zone (red) for (A) high $\mathrm{P}_{\mathrm{TP}}$ and (B) low $\mathrm{P}_{\mathrm{TP}}$. Upper horizontal line $=\mathrm{P}_{\mathrm{TP}}$, lower horizontal line $=\mathrm{P}_{\text {det }}$.
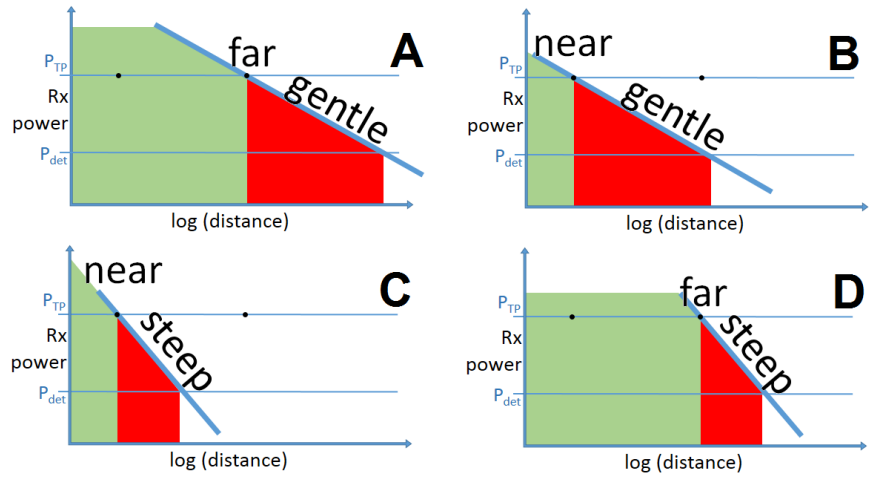

Fig. 5: Coverage zone (green) and interference zone (red) for (A) far intercept and low slope, (B) near intercept and low slope, (C) near intercept and high slope, and (D) far intercept and high slope. Upper horizontal line $=\mathrm{P}_{\mathrm{TP}}$ (incl. margins), lower horizontal line $=\mathrm{P}_{\text {det }}$.

accounted for in the network planning. The site survey is an experimental process with results depending on the specific location (spatial) and specific moment (temporal) of the measurement. On average over the entire environment, the power measured with the handheld device is therefore a median value at each location and thus corresponds to not using any temporal fading margin (vs. margin of $5 \mathrm{~dB}$ for the automated planning). Similarly, due to the use of a shadowing margin in the automated network planning, the assumed coverage area is explicitly reduced. Hence, the automated planning will be more robust to signal fades, but requires more APs. On the other hand, the planning algorithm applies an extrapolation of the constructed model to an entire environment, providing less certainty on the accuracy when comparing to a site survey with actual measurements. In the considered environment, which is quite homogeneous (open environment where all racks are similar), the automated planning will likely be accurate. However, in more heterogeneous environments with many different object types, the automated planning may be less suitable, due to the constructed path loss model not being generally applicable (see Table I, 'applicability').

With respect to the required effort, the automated planning process obviously has benefits over the site survey. The site survey had to be executed at night in order not to disturb the warehouse operators and took three weeks. The automated network planning took about 1 day to perform the measurements for the creation of the path-loss model. The total execution time of the network planning process is several days but does not require any substantial human effort (see Table I, 'cost'). In any case, the obtained deployment shows that in large environments, a lot of APs will be needed. Hence, the issue of intra-network interference arises. Although the network is designed to achieve a certain throughput, the presence of coexisting APs operating on the same frequency will reduce the actual throughput. The next section will shortly link these issues to the scenario that is considered in this work.

TABLE I: Results and characteristics of automated WHIPP network planning vs. experimental site survey planning.

\begin{tabular}{|c|c|c|}
\hline & WHIPP & Site survey \\
\hline \multicolumn{3}{|l|}{ Results } \\
\hline$\#$ access points & 92 & 73 \\
\hline \multicolumn{3}{|l|}{ Characteristics } \\
\hline $\begin{array}{r}\text { accuracy } \\
\text { applicability } \\
\text { cost } \\
\text { interference assessment }\end{array}$ & $\begin{array}{r}\text { high } \\
\text { homogeneous } \\
\text { low } \\
\text { easy }\end{array}$ & $\begin{array}{r}\text { very high } \\
\text { general } \\
\text { high } \\
\text { difficult }\end{array}$ \\
\hline
\end{tabular}

\section{B. Intra-network interference}

In the considered warehouse scenario with automated planning, a high number of APs (92) is needed, due to the high $\mathrm{P}_{\mathrm{TP}}(-68 \mathrm{dBm})$, and the high sum of shadowing and fading margin (almost $9 \mathrm{~dB}$ ). Fig. 6 shows the warehouse, to which the frequency planning algorithm of [5] was applied. Still, up to 31 APs work on the same frequency.

At the random location indicated with the orange star, a wireless device is connected to the light blue AP right next to it. The zone enclosed by the green line is the zone where APs will cause, at the indicated location, a median power higher than the throughput power threshold $(>-68 \mathrm{dBm})$. The zone enclosed by the red line roughly shows all AP locations that cause, at the indicated location, a median power that is higher than the assumed detection threshold of $-95 \mathrm{dBm}$. When all 92 APs operate on the same channel, this amount is 36 APs (plus the AP to which the user is connected); with the frequency planning algorithm applied, this number reduces to 13 interfering APs. Although frequency planning obviously reduces the number of interfering APs by almost a factor 3 (here 2.77), these results show that interference issues are still likely to appear. Fig. 6 shows that the coverage range of an AP is very small (especially when accounting for margins, blue area in Fig. 6), compared to its interference range (red area in Fig. 6). This is especially the case in the direction of the racks' orientation. The large difference between the coverage zone and interference zone is due to the high throughput power threshold and link budget margins. Compared to a site survey, the use of an automated planner easily allows identifying possibly interference problems. In a site survey, logging interference powers from many APs would result in an enormous amount of work (see Table I, 'interference assessment'). Given the large number of deployed APs due to the high throughput power threshold, it might turn out 


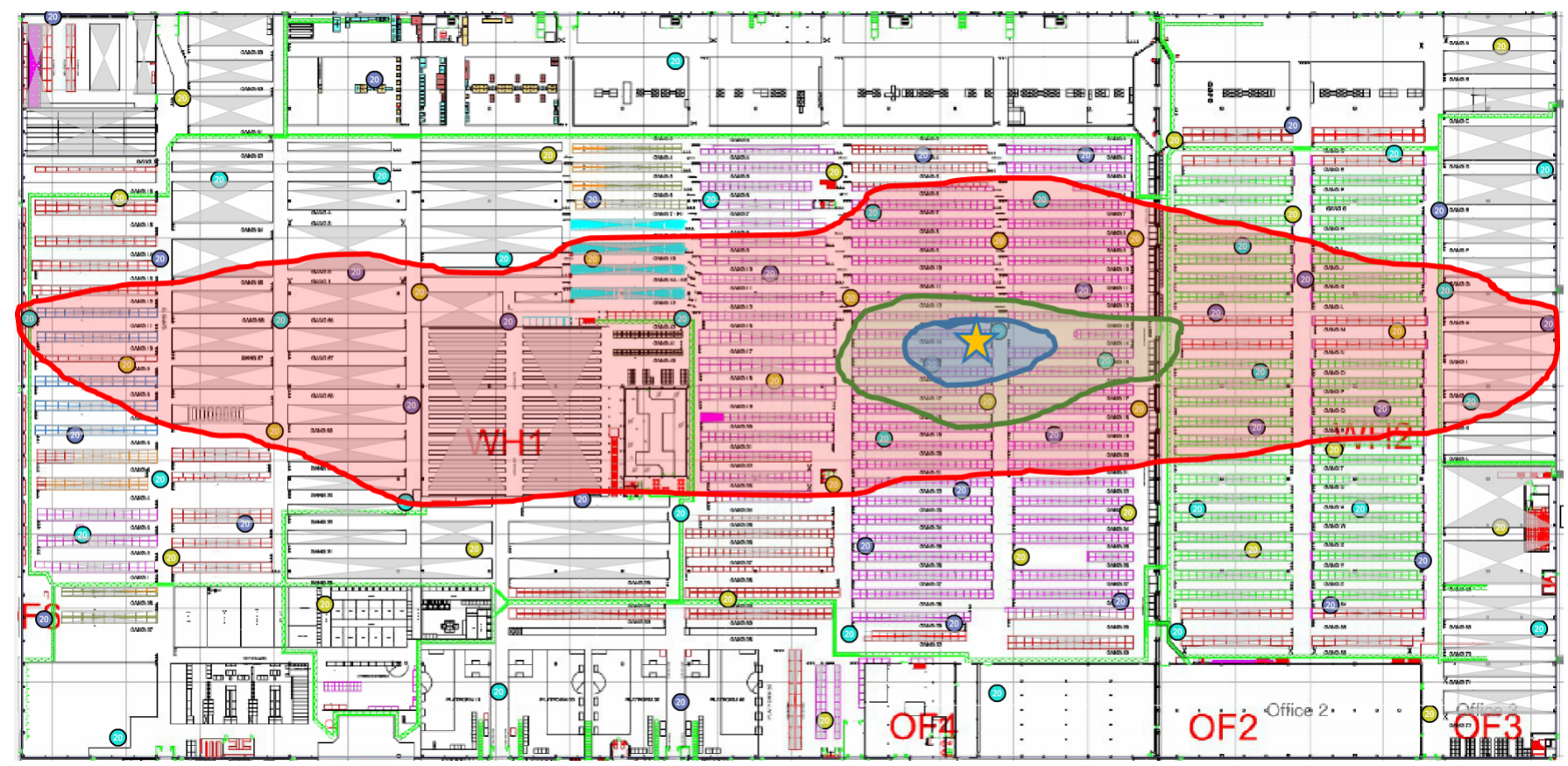

Fig. 6: Map of warehouse environment $(415 \mathrm{~m}$ x $200 \mathrm{~m})$ with indication of APs for which, based on the median received power, the wireless device at the location of the orange star is in the interference zone (enclosed by red outer line), in the coverage zone without accounting for margins (enclosed by green line), and in the coverage zone accounting for margins (enclosed by blue inner line). APs are indicated with coloured dots, where different colours indicate different operating channels.

beneficial to plan the network for a lower throughput or to use smaller margins.

\section{CONCLUSION}

A WiFi network planning process has been conducted in a very large industrial warehouse. The output of an automated software planner and a site survey yielded a comparable number and location of access points. Differences can be attributed to the assumption of a more strict link budget (e.g., shadowing and fading margin) in the network planner. Although a site survey should inherently guarantee the performance of the resulting network, its execution is much more expensive than an automated planning (three weeks vs. one day in this case). Moreover, in industrial environments, site surveys often have to be executed at night in order not to disturb the normal warehouse operation. In order to obtain a fully reliable network planning at the lowest cost, it could be therefore advisable to combine an automated network planning with a limited validation site survey. An assessment of the powers received from the different access points shows that the interference zone of the access points is much larger than the coverage zone, which is caused by the high throughput requirements. The results clearly indicate that future research is needed on how network planning in large environments is impacted by intra-network interference. An assessment of the influence of different throughput power thresholds on intra-network interference will be executed in different environments (with different path loss models or the power decays) and measurements of the actual throughput under the presence of interference will be performed. Currently, no wireless network planners are available that accurately deal with this issue.

\section{ACKNOWLEDGMENT}

This work was supported by FORWARD, a project cofunded by iMinds, a digital research institute founded by the Flemish Government. Project partners are ArcelorMittal, Egemin, Excentis, Siemens, and Volvo, with project support from IWT. E. Tanghe is a Post-Doctoral Fellow of the FWO-V (Research Foundation-Flanders).

\section{REFERENCES}

[1] E. Tanghe, W. Joseph, L. Verloock, L. Martens, H. Capoen, K. Van Herwegen, and W. Vantomme, "The industrial indoor channel: large-scale and temporal fading at 900, 2400, and $5200 \mathrm{mhz}$," Wireless Communications, IEEE Transactions on, vol. 7, no. 7, pp. 2740-2751, July 2008.

[2] D. Plets, W. Joseph, K. Vanhecke, E. Tanghe, and L. Martens, "Coverage Prediction and Optimization Algorithms for Indoor Environments," EURASIP Journal on Wireless Communications and Networking, Special Issue on Radio Propagation, Channel Modeling, and Wireless, Channel Simulation Tools for Heterogeneous Networking Evaluation, vol. 1, 2012. [Online]. Available: http://jwcn.eurasipjournals.com/content/2012/1/123

[3] Q. Li, M. Ho, V. Erceg, A. Janganntham, and N. Tal, "802.11n channel model validation," IEEE 802.11-03/894r1, 11-03-0894-01-000n-802-11nchannel-model-validation.pdf," Tech. Rep., 2003.

[4] D. Plets, W. Joseph, K. Vanhecke, and L. Martens, "Exposure Optimization in Indoor Wireless Networks by Heuristic Network Planning," Progress In Electromagnetic Research (PIER), vol. 139, pp. 445-478, 2013.

[5] N. Liu, D. Plets, W. Joseph, and L. Martens, "An algorithm for optimal network planning and frequency channel assignment in indoor wlans," in IEEE Antennas and Propagation Society International Symposium. IEEE, 2014, pp. 1177-1178. 